\title{
Exploring Filipino adolescents' conception of happiness
}

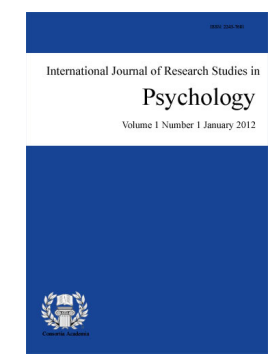

ISSN: 2243-7681 Online ISSN: 2243-769X

OPEN ACCESS

\section{Abstract}

The current paper aimed to explore the meaning and conception of happiness among selected Filipino adolescents. Ten Filipino college students whose ages range between 16 and 20 were selected through purposive sampling. The study employed qualitative-descriptive research design. Findings showed that happiness is a multifaceted construct that involved the following major themes: satisfaction of wants, absence of worries, expression of positive emotions, motivational drive, and fulfillment of relational needs. Distinct personal characteristics and positive behavioral strategies predictive of happiness were identified as well. Enhancing positive emotions, positive traits and behavioral strategies of individuals from this developmental phase may be a good step to increase their social, psychological and occupational functioning in the form of academic, extra-curricular, co-curricular endeavours.

Keywords: behavioral strategies; Filipino adolescents; happiness; positive psychology; subjective well-being 


\section{Exploring Filipino adolescents' conception of happiness}

\section{Introduction}

Happiness has been prevalent in researches concerning positive psychology. It endeavors to zero in on positive emotions, traits and institutions as substrates of psychological wellness as opposed to the alleviation of negative symptoms in the traditional psychopathology model. Beneficial consequences of living a happy life tend to be a substantial factor in the emergence of this empirical inquiry (Primasari \& Yuniarti, 2010). Diener, Lyubomirsky and King (2005) asserted that happiness can be directly related to success. As people attain success, they tend to have positive affect which leads to happiness. That hypothesis was similar to the findings of Heady \& Veehoven (as cited in Primasari \& Yuniarti, 2010) which capitalized on the influence of happiness to the feeling of satisfaction. What remained constant across different studies is the formulation that happiness is an antecedent, correlate and predictor of positive psychological outcomes (Parks, Della Porta, Pierce, Zilca, \& Lyubomirsky, 2012; Lyubomirsky, Diener, \& King, 2005). To better understand the dynamics of one's subjective state of well-being, empirical studies were created using quantitative designs (Malkoc, 2011; Toner, Haslam, Robinson \& Williams, 2012; Parks, Della Porta, Pierce, Zilca, \& Lyubomirsky, 2012; Tkach \& Lyubomirsky, 2006; Ganesan \& Reyes, 2011) and qualitative designs (Chiu, Hsieh, Yeh, Kuo, Lee, \& Yu, 2010; Lu \& Gilmour, 2004).

Several studies have investigated happiness using quantitative research designs (Malkoc, 2011; Toner, Haslam, Robinson \& Williams, 2012; Parks, Della Porta, Pierce, Zilca, \& Lyubomirsky, 2012; Tkach \& Lyubomirsky, 2006; Ganesan \& Reyes, 2011). As early as 1960's, happiness was being explored through the use of different survey forms (Argyle as cited in Primasari \& Yuniarti, 2010). Some of them focused on possible predictors of happiness which majority fall into the result of social economic status of a person (Kozaryn, 2010). On the contrary, Piccolo, Judge, Takahash, Watanabe, and Locke (2005), claimed that positive self-regard is the most important predictor of happiness and as well as life satisfaction. Using the Big Five Personality Model, Tkach, and Lyubomirsky (2006) found out that personality traits can mediate happiness, especially extraversion. Howell, Chenot, Hill, and Howell (2011) concluded that satisfaction of daily psychological needs results to happiness. As children mainly focuses on their peers and family relationship (Holder \& Coleman, 2007) as similar with the adolescents (Chen \& Lu, 2009) and romantic relationship for married adults (Demir, 2008; Demir, 2010) while best friend and mother relationship arouse for single adults (Demir, 2010).

Qualitative research designs were also employed to strengthen formulations on happiness (Chiu, Hsieh, Yeh, Kuo, Lee, \& Yu, 2010; Lu \& Gilmour, 2004). For instance, Chiu, Hsieh, Yeh, Kuo, Lee, and Yu (2010) explored conception of happiness among Chinese students through a model-building approach. They have identified specific determinants of happiness, specifically caring, environment, balancing, owning, playing, being rewarded, interaction, and self-realization. Lu and Gilmour (2004) utilized a qualitative design as part of their two-fold study in figuring out conceptions of happiness between individuals from Asian and Western contexts. Findings of their study validated the underpinning that conception of happiness among Asian people is socially-construed as opposed to individually-construed well-being of Euro-American individuals.

Regardless of the type of research design employed, consistencies are demarcated from previous studies. Happiness was framed in the context of specific theoretical frameworks like the subjective well-being (SWB) model, psychological well-being (PWB), self-determination theory, trait theory among others. Despite distinctions among the premises of such theories, three general predictors of happiness are identified which include: 1.) life circumstances and demographics, 2.) traits and dispositions, and 3.) intentional behaviors (Tkach \& Lyubomirsky, 2006).

As an attempt to further strengthen empirical utility of the dispositional and situational determinants of happiness, Tkach and Lyubomirsky (2006) formulated a study that highlighted personal characteristics and 
happiness increasing strategies of students from United States of America. Behavioral strategies involved mental control, goal pursuit, affiliation, active leisure, passive leisure, partying, direct attempts and religion. These findings augmented our knowledge of how dynamics of happiness can be observed from people in individualistic societies like USA. However, given that people from collectivistic societies reward relationships more than individually-pertinent variables such as self-esteem and personal achievement, it is possible to draw a different conception and behavioral manifestation of happiness (Chiu, Hsieh, Yeh, Kuo, Lee, \& Yu, 2010; Diener \& Diener, 1995). Cultural differences essentially accounts for the variances on how psychological processes like happiness can be conceptualized between Western and Asian people (Markus \& Kitayama, 1998). In effect, there is an imminent necessity to advance empirical inquiries concerning culturally-sensitive conceptions of happiness.

To date, few studies have been generated to look at conception of happiness in the context of collectivistic nations. For example, Lu and Gilmour (2004) hypothesized attainment of a positive and joyous state among Chinese college students. In particular, they posited the assertion that happiness of Asian individuals is socially-oriented, in contrast with the individually-oriented happiness of Western people (i.e. Euro-American). That said, it can be captured that social variables such as social support (Ganesan \& Reyes, 2011), interdependent self-construal (Reyes, 2005) are necessary elements in understanding happiness in the context of Asian societies. In spite of these developments in happiness researches in the Asian region, there is still a dearth of literature that delves on its conception.

\subsection{The Present Study}

The first aim of the study is to look at how Filipino adolescents define happiness as meaning and concepts about a specific phenomenon is highly shaped by one's culture (Bruner, 1990). In other words, we take the stance that conception of happiness among Asians significantly differs from that of Westerners. The second objective is to explicitly examine personal characteristics that describe a happy Filipino adolescent. Given that individuals under this stage are prone to experience wide array of life challenges as a result of their quest to establish a stable sense of identity, it is quite interesting to look at specific features that typifies positive and joyous state sensitive to their developmental phase. The need to specify individual characteristics can be demarcated as well on the fact that individuals in the Asian cultures have lower regards on self-esteem and self-consistency in predicting psychological outcomes as opposed to higher levels of such variables from people in individualistic societies (Markus \& Kitayama, 1998). The third objective is to identify particular behavioral strategies that are predictive of happiness among the intended participants since intentional behaviors account for a significant portion of how happiness can be realized among individuals (Tkach \& Lyubomirsky, 2006). The ultimate goal of the study is to better understand conception of happiness among selected Filipino adolescents.

\section{Methods}

\subsection{Research Design}

The study employed qualitative-descriptive research design. Qualitative research is " $a$ form of systematic empirical inquiry into meaning" (Shank, 2002, p. 5). In the present paper, definition of happiness, personal characteristics predictive of happiness and positive behavioral strategies of selected Filipino adolescents are examined through individual interviews.

\subsection{Participants}

Ten Filipino adolescents whose age range from 16 to 20 were selected through purposive sampling technique. Inclusion criteria involved: a.) age should range from 15 to 21 ; b.) must be enrolled in collegiate institution; and c.) free from serious psychological condition. Prior to the data collection, participants were informed about the nature, benefits and risks in joining the study through informed consent. This is to ensure ethical considerations are met in the conduct of the study. 


\subsection{Research Instruments}

The current research utilized semi-structured interviews as a way to collect responses from the participants. A duly-validated interview guide question provided the specific structure for the execution of the data collection procedure. Some of the sample questions include; a.) How do you define happiness? and b.) What are the characteristics of a happy person? Individual interview sessions were recorded through a digital recorder. The mean length of the interviews is 35 minutes.

\subsection{Data Analysis}

After recordings and transcribing the interview sessions, these were analyzed for specific themes. Common themes were analyzed to capture the conception of happiness of the participants regarding a positive and joyous state. Personal characteristics and behavioral strategies that typify happy individuals were enumerated as well.

\section{Results and Discussions}

The section presents the results of the current investigative inquiry. Major themes generated from the interviews are integrated in the discussion. It starts with the definition of happiness. It identifies personal characteristics that typify a happy a Filipino adolescent. It also presents specific behavioral strategies employed by the participants to be happy in their respective lives. Reporting of the responses partially follows how findings in qualitative-descriptive data are presented through the use of tables expressing percentage of responses and corresponding qualitative interpretations.

\subsection{Definition of Happiness}

\section{Happiness as Satisfaction of Wants}

Most of the participants viewed happiness as a state of contentment resulting from attainment of aspired tangible and intangible things. They believed that happiness is a positive evaluation of their lives. Achieving personal goals and aspirations, therefore, is a highly influential element to be happy. As one participant averred: "I'm just a simple person, as long as I'm getting things I would like to get, I will be happy". Another participant shared: "Happiness for me is being able to know things I love to do and to achieve things I aspired to have". Others reiterated on the viewpoint that happiness is a feeling of fulfillment that stemmed from satisfaction of needs and wants. Conception of happiness as a satisfaction of wants showed consistency with formulations from previous studies and theories (Lu \& Gilmour, 2004; Tkach \& Lyubomirsky, 2006; Primasari \& Yuniarti, 2010).

\section{Happiness as Absence of Worries}

The conceptual understanding that happiness is characterized by absence of negative emotions was also evident in the participants' responses. They defined happiness in a different perspective by highlighting on the role of reduced negative affect as opposed to the enhancement of their positive joyous states. Negative emotions encompass feeling of sadness, anxiety and confusion. Consistent with this definition, one participant verbalized: "Happiness for me is when there are just few things I have to worry about". Likewise, another adolescent shared: "I'm happy whenever I'm not facing any serious problem". Though this seems to contradict the usual way we look at happiness, it is still a significant conception given that negative affect is a core element in other well-being frameworks (Lu \& Gilmour, 2004; Tkach \& Lyubomirsky, 2006).

\section{Happiness as Expression of Positive Emotions}

As conceived by the participants, happiness is an act of showing pleasant emotions. Smiling and laughing are commonly identified behavioral manifestations of a joyous state. Positive affect is a core element in their happiness. To support this major theme, a participant mentioned: "I can say that I'm happy whenever I used to smile a lot". Another participant said: We can say that people are happy when they are laughing". This view 
exhibits parallelism with the way Chinese college students define happiness (Lu \& Gilmour, 2004).

\section{Happiness as a Motivational Drive}

Surprisingly, most of the participants defined happiness as an intrinsic impetus to perform things or endeavors that they used to engage in. Happiness serves as a motivating force to keep them from working on the tasks they are confronted with. This is a distinct way to describe happiness as previous studies emphasized more on the notion that happiness is a supreme and ultimate goal to one's existence. As shared by one participant: "For me, I t serves as the source of strength and vigor that keeps me from focusing on the things I'm supposed to do". In addition, another participant said: "I get to accomplish a lot of things whenever I'm happy". While most of the studies highlighted on happiness as an outcome, results of the study depicted that it is a process element predictive of positive behavioral outcomes.

\section{Happiness as Fulfillment of Relational Needs}

The conception of happiness as a satisfaction of needs to interact with social support system (e.g. primary, secondary) is reinforced in the study. Feeling of contentment and joy resulting from meaningful relationships is a central theme that can be found out from the participants' responses. The most crucial relationships emerge from family, friends, peers and colleagues. For example, one participant said: "Whenever I see and keep in touch with my parents and my friends, I feel glad and happy". Most of them shared commonalities in terms of prioritizing the impact of relationship satisfaction on the achievement of a positive and joyous state. This conception is highly relevant to the participants at hand since individuals from collectivistic societies may show a greater likeliness to view themselves in the context of socially-meaningful relationships (Markus \& Kitayama, 1998). Given that interacting with others is highly rewarded in the Philippine culture (e.g. closely-knit family structure), behaviors that typify it may predispose them to experience a positive and joyous emotional state. Similar definition of happiness was seen from Chinese college students (Lu \& Gilmour, 2004).

\subsection{Personal Characteristics of a Happy Person}

The participants were asked to describe specific personal characteristics that best represents a typical happy Filipino adolescent. The salient personal indicators of happiness are presented in the table below.

\section{Table 1}

Personal Characteristics of Happy Filipino Adolescents

\begin{tabular}{ccc}
\hline Personal Characteristics & $F$ & $\%$ \\
\hline Being worry-free & 4 & 40 \\
Being helpful & 10 & 100 \\
Being optimistic & 6 & 60 \\
Being reflective & 2 & 20 \\
Being creative & 2 & 20 \\
Being simple & 2 & 20 \\
Being jolly & 8 & 80 \\
Being God-centered & 4 & 40 \\
\hline
\end{tabular}

Participants highlighted being helpful as the most robust personal characteristic of happy Filipino adolescents. It implies that the desire to help others is necessary to attain a positive and joyous emotional state. This also means that inclinations to show genuine altruistic concern to other people are predictive of happiness. Being jolly appeared to be as another prevalent personal characteristic that typified happy Filipino adolescents. That said, happy Filipino adolescents are perceived to be as fun-loving and cheerful despite challenges they are confronted with. This personal characteristic also validates the conception of happiness as expression of positive emotions. Being worry-free was also expressed as a characteristic predictive of happiness which supported the major theme of happiness as absence of worries. Being reflective, creative and simple, on the other hand, were personal characteristics seldom seen from the participants at hand. Another contextually-relevant personal 
feature seen among them is being God-centered. This personal quality may be attributed to the fact that majority of individuals living in the Philippine society are Christians.

\subsection{Happiness-Increasing Strategies}

The participants were asked to share particular behaviors they engage in to be happy. Most of these strategies are interpersonal in nature although some of them are intrapersonal. The frequently occurring positive behavioral strategies are listed below.

Table 2

Positive Behavioral Strategies of Happy Filipino Adolescents

\begin{tabular}{lcc}
\hline \multicolumn{1}{c}{ Positive Behavioral Strategies } & $F$ & $\%$ \\
\hline Helping others & 10 & 100 \\
Playing computer games & 2 & 20 \\
Frequent smiling or laughing & 6 & 60 \\
Making other people happy & 4 & 40 \\
Serving the Church & 2 & 20 \\
Listening to music & 2 & 20 \\
Avoiding negative thoughts & 4 & 40 \\
Competing with one's self & 2 & 20 \\
Joining sports activities & 2 & 20 \\
Interacting with family, friends and significant others & 10 & 100 \\
\hline
\end{tabular}

Evidently, participants engaged more in socially-oriented strategies to enhance their subjective state of psychological wellness. Specifically, helping others, interacting with family, friends and significant others and making other people happy are specific tasks that they commonly employ to be happy. Helping others appeared to be associated to happiness as altruism which is one of the character strengths virtues cited by Martin Seligman. These two happiness-increasing behaviours were consistently seen in the study of Tkach and Lyubomirsky (2006) that hypothesized these strategies under social affiliation. It also validates the premises from past theoretical and empirical underpinnings that socially-engaging endeavours enhance happiness (Lu \& Gilmour, 2004; Lu \& Shih, 1997; Csikszentmihalyi \& Hunter, 2003).

On the other hand, intrapersonal behavioral strategies were also utilized by participants to be happy. In particular, frequent smiling serves as one of the ways to optimize their positive and joyous state. Given that expression of positive emotions is a central element on their conception of happiness, this endeavor is a significant behavioral indicator of happiness. Other individualistic strategies they employ to become happy include competing with their selves, playing computer games and listening to music. Competing with their selves is strategy that involves using one's self as a frame of reference in performing tasks that require competition in the class and other similar situations. What makes this finding interesting is the fact that these participants came from collectivistic societies where socially-engaging behaviors are commonly linked to positive psychological outcomes. However, it may be inappropriate to infer that Filipino adolescents are more likely to manifest socially-oriented strategies to be happy as influenced by greater susceptibility to interdependent construal (Reyes, 2005). Probably, participants' engagement in individually-oriented strategies can be accounted to their developmental phase (psychosocial stage of identity versus role confusion) where establishment of identity is a crucial task to deal with. Competing with one's self may be a more culturally-rewarding strategy since it reduces the chance of having conflict with other people.

Playing computer games and listening to music were found out to be behavioral engagements predictive of happiness. These are forms of passive leisure strategies identified in the empirical inquiry of Tkach and Lyubomirsky (2006). Yet, passive leisure strategies were not significantly linked to happiness as proven by past studies (Erber as cited in Tkach \& Lyubomirsky, 2006). Possibly, differences in the cultural orientation of Filipino and Western adolescents may explain the inconsistency on the result. 


\subsection{Filipino adolescents' conception of happiness}

As we illustrated in the elaboration of the findings, Filipino adolescents' understanding of happiness revolves around expression of positive emotions, absence of negative affect, relational needs fulfillment, wants satisfaction, and motivation. Such elements are central in conceptualizing well-being of Filipino adolescents. From the major themes that best represent their inclinations towards positive and joyous emotional state, several inferences can lead us towards crafting a better conception of how individuals from this developmental phase become happy.

The current study found out that adolescents' way of defining happiness has cognitive and affective components. It validates the core dimensions of the Subjective Well-Being (SWB) theory on happiness by Diener (1984). In the SWB model, satisfaction with life resembles satisfaction of wants while positive and negative affects typify positive emotions and absence of worries in the present investigative inquiry. Happiness is seen as a construct that is characterized by presence of positive emotions, absence of negative emotions and positive appraisal of life. Given the resemblance between the core themes of happiness recognized in the study and the dimensions of SWB, pragmatic utility of the latter framework in the Philippine context can be further enhanced.

Happiness has a motivational value that is predictive of positive psychological and behavioral outcomes. It highlights the role of joyous state on the attainment of various success measures as opposed to the conception of happiness as an outcome (Primasari \& Yuniarti, 2010). Happy adolescents, therefore, may manifest greater likeliness of getting better academic performance or endeavors that would require them to execute tasks in purposeful and meaningful ways. It implies that positive emotions play a crucial function in motivating adolescents to exert greater efforts in attaining specific goals in life. Achieving a pleasant emotional state is a significant way to optimize opportunities for greater success in life. This is a unique definition as it endorsed happiness as an essential substrate towards achieving life satisfaction more than a positive outcome.

The definition of happiness as a fulfillment of relational needs suggests that the way Filipino adolescents view happiness may be socially-construed. Achievement of a positive and joyous state is a phenomenon which may be optimized through performance of socially-engaging behaviors. This conception is consistent with Lu and Gilmour's (2004) premise that happiness of Asian individuals like Chinese are socially-oriented as opposed to individualistic orientations of Western individuals. To enhance well-being, it is important to consider social entities that may be predictive of life satisfaction, especially in the Philippine context where social-connectedness is well-reinforced more than individually-oriented constructs.

Distinct personal attributes characterize Filipino adolescents who are happy (e.g. helpful, optimistic, reflective, God-centered, etcetera). Yet, being helpful and jolly appeared to be the salient traits that best represent happy individuals from this developmental stage. These happiness-increasing traits serve as dispositional elements that further strengthen their joyous states since showing altruistic behaviors is highly rewarded in collectivistic societies like the Philippines. Given that happiness of Asian individuals are socially-oriented, possessing individual characteristics that foster social growth and connectedness like being altruistic and jolly are influential to establish meaningful relationships and fulfill their relational needs. Consequently, their inclination to a positive and joyous state may be enhanced.

Findings of the current study generated specific insights regarding Filipino adolescents' conception of happiness. In spite of its theoretical, empirical and practical significance, certain limitations may be drawn. For instance, the number of participants is relatively small $(\mathrm{n}=10)$ which poses restrictions on its applicability to the general population. Given that it was found out in the study that happiness has a motivational drive and relational need fulfillment domains that optimizes success measures, future researchers are recommended to look at specific paths by which happiness can motivate adolescents to become more successful on certain aspects of 
their lives through examining specific social units that better account for their propensity to become happy in their lives.

\section{Conclusion}

The study investigated conception of happiness among Filipino adolescents. It explores definition of happiness along with positive personal qualities and behavioral strategies. From the findings of the study, the applicability of the SWB Model of Diener (1984) may be realized as the conceptual themes resembled three core elements in the SWB's conceptualization of psychological wellness (e.g. positive affect, negative affect and life satisfaction). Happiness is a construct that has cognitive, affective and behavioral dimensions. It served as a psychological impetus as well towards specific measures of accomplishment. Though, it can be surmised that their definition of happiness may be highly shaped by a need to engage in socially-meaningful relationships. This may pose a significant implication on the way psychologists conceptualize happiness as the positive affect, negative affect, and life satisfaction domains of well-being may be dependent on the way these individuals establish interpersonal connections with other people as opposed to the desire of individuals from Western societies to thrive for personal autonomy or independence. Hence, promotion of counseling interventions that enhance well-being must be parallel with these conceptions to yield positive and desirable psychological outcomes.

\section{References:}

Bruner, J. (1990). Acts of meaning. Cambridge, MA: Harvard University Press.

Chiu, H., Hsieh, Y., Yeh, H., Kuo, C., Lee, M., \& Yu, D. (2011). The connection between happiness and service business: A preliminary study. Journal of Happiness Studies, 12, 841-860. http://dx.doi.org/10.1007/s10902-010-9230-x

Csikszentmihalyi, M., \& Hunter, J. (2003). Happiness in everyday life: The use of experience sampling. Journal of Happiness Studies, 4, 185-199. http://dx.doi.org/10.1023/A:1024409732742

Demir, M. (2008). Sweetheart, you really make me happy: Romantic relationship quality and personality as predictors of happiness among emerging adults. Journal of Happiness Studies, 9(2), 257-277. http://dx.doi.org/10.1007/s10902-007-9051-8

Demir, M. (2010). Close relationships and happiness among emerging adults. Journal of Happiness Studies, 11(3), 293-313. http://dx.doi.org/10.1007/s10902-009-9141-x

Diener, E., \& Diener, M. (1995). Cross-cultural correlates of life satisfaction and self-esteem. Journal of Personality and Social Psychology, 68, 653-663. http://dx.doi.org/10.1037/0022-3514.68.4.653

Diener, E. (1984). Subjective well-being. Psychological Bulletin, 95, 542-575. http://dx.doi.org/10.1037/0033-2909.95.3.542

Ganesan, A., \& Reyes, J.A.S. (2011). Factors predictive of the subjective well-being among Malaysian ethnic minorities, Philippine Journal of Counseling Psychology, 13(1), 15-28.

Hoellerer, N. (2011). The use of quantitative and ethnographic research to enhance the measurement and operationalisation of gross national happiness. Journal of Bhutan Studies, 26-54.

Howell, R., Chenot, D., Hill, G., \& Howell, C. (2011). Momentary happiness: The role of psychological need satisfaction. Journal of Happiness Studies, 12(1), 2-15. http://dx.doi.org/10.1007/s10902-009-9166-1

Kozaryn, A. (2010). Europeans work to live and Americans live to work (Who is happy to work more: Americans or Europeans?) Journal of Happiness Studies, 12(2), 225-243. http://dx.doi.org/10.1007/s10902-010-9188-8

Lu, L., \& Gilmour, R. (2004). Culture and conceptions of happiness: Individual and social oriented SWB. Journal of Happiness Studies, 5, 269-291. http://dx.doi.org/10.1007/s10902-004-8789-5

Lu, L., \& Shih, J.B. (1997). Sources of happiness: A qualitative approach. The Journal of Social Psychology, 137(2), 181-187. http://dx.doi.org/10.1080/00224549709595429

Lyubomirsky, S., King, L., \& Diener, E. (2005). The benefits of frequent positive affect: Does happiness lead to 
success? Psychological Bulletin, 131, 803-855. http://dx.doi.org/10.1037/0033-2909.131.6.803

Malkoc, A. (2011). Big five personality traits and coping styles predict subjective well-being: A study with a Turkish sample. Procedia Social and Behavioral Sciences, 12, 577-581. http://dx.doi.org/10.1016/j.sbspro.2011.02.070

Markus, H. R., \& Kitayama, S. (1998). The cultural psychology of personality. Journal of Cross-Cultural Psychology, 29, 63-87. http://dx.doi.org/10.1177/0022022198291004

Ott, J. C. (2011). Limited experienced happiness or unlimited expected utility, What about the differences? Journal of Happiness Study, 12(3), 519-524. http://dx.doi.org/10.1007/s10902-010-9216-8

Parks, A. C., Della Porta, M. D., Pierce, R. S., Zilca, R., \& Lyubomirsky, S. (2012, May 28). Pursuing happiness in everyday life: The characteristics and behaviors of online happiness seekers. Emotion. Advance online publication. http://dx.doi.org/10.1037/a0028587

Piccolo, R., Judge, T., Takahash, K., Watanabe, N., \& Locke, E. (2005). Core selves-evaluation in Japan: Relative effects on job satisfaction, life satisfaction and happiness. Journal of Organizational Behavior, 965-984.

Primasari, A., \& Yuniarti, K. W. (2012). What make teenagers happy? An exploratory study using indigenous psychology approach. International Journal of Research Studies in Psychology, 53-61.

Reyes, J. A. S. (2005). Conceptualizing the Happy Filipino: Implicit theories of counselors and clients. Philippine Journal of Counseling Psychology, 7(1), 99-113.

Shank, G. (2002). Qualitative Research. A personal skills approach. New Jersey: Merril Prentice Hall.

Tkach, C., \& Lyubomirsky, S. (2006). How do people pursue happiness? Relating personality, happiness-increasing strategies, and well-being, Journal of Happiness Studies, 7, 183-225. http://dx.doi.org/10.1007/s10902-005-4754-1

Toner, E., Haslam, N., Robinson, J., \& Williams, P. (2012). Character strengths and well-being in adolescence: Structures and correlates of the Values in Action Inventory of strengths for children. Personality and Individual Differences, 52, 637-642. http://dx.doi.org/10.1016/j.paid.2011.12.014 
Datu, J. A. \& Valdez, J. P. 